\title{
COMPLETENESS THEOREM FOR SINGULAR BIPROBABILITY MODELS
}

\author{
MIODRAG RAŠKOVIĆ
}

(Communicated by Thomas J. Jech)

\begin{abstract}
The aim of the paper is to prove the completeness theorem for singular biprobability models. This also solves Keisler's Problem 5.4 in the singular case (see [3]). The case of absolute continuity is considered in [6].
\end{abstract}

Let $A$ be a countable admissible set and $\omega \in A$. The logic $L_{P_{1} P_{2} A}$ is similar to the standard probability logic $L_{A P}$. The only difference is that two types of probability quantifiers $\left(P_{1} \vec{x} \geq r\right.$ and $\left.P_{2} \vec{x} \geq r\right)$ are allowed.

The probability logic $L_{A P}$ was introduced in [2] by H. J. Keisler. It is the logic which has formulas like those of $L_{A} \subseteq L_{\omega_{1} \omega}$, except that quantifiers $P \vec{x} \geq r(\vec{x}$ is a finite sequence of variables, $r \in[0,1]$ a real number) are used instead of the usual $\forall x$ and $\exists x$. A model is a probability space with measurable relations with respect to some extension of product measure. A formula $(P \vec{x} \geq r) \varphi(\vec{x})$ is true in the model if $\{\vec{x}: \varphi(\vec{x})\}$ has probability greater than $r$.

A singular biprobability model is a structure $\left(\mathfrak{A}, \mu_{1}, \mu_{2}\right)$ where

$$
\mathfrak{A}=\left(A, R_{i}, c_{j}\right)_{i \in I, j \in J}
$$

is a classical structure without operations and $\mu_{1}, \mu_{2}$ are types of probability measures such that $\mu_{1}$ is singular with respect to $\mu_{2}$, i.e. $\mu_{1} \perp \mu_{2}$.

The quantifiers are interpreted in the natural way i.e.

$$
\left(\mathfrak{A}, \mu_{1}, \mu_{2}\right) \vDash\left(P_{1} \vec{x} \geq r\right) \varphi(\vec{x}) \quad \text { iff } \mu_{1}^{(n)}\left\{\vec{x} \in A^{n}:\left(\mathfrak{A}, \mu_{1}, \mu_{2}\right) \vDash \varphi(\vec{x})\right\} \geq r
$$

for $i=1,2$. (The measure $\mu_{i}^{(n)}$ is the restriction of the completion of $\mu_{i}^{n}$ to the $\sigma$-algebra generated by the measurable rectangles and the diagonal sets $\{\vec{x} \in$ $\left.A^{n}: x_{i}=x_{j}\right\}$.)

Axioms and rules of inference are those of $L_{\mathscr{A} P}$, as listed in [1] in conjunction with the axiom $B_{4}$ from [3] with the remark that both $P_{1}$ and $P_{2}$ can play the role of $P$, together with the following axioms:

Axioms of continuity.

$$
\begin{aligned}
& \bigwedge_{n \geq 1} \bigvee_{m \geq 1}\left(P_{i} \vec{y}<\frac{1}{n}\right)\left(P_{j} \vec{x} \in\left[r-\frac{1}{m}, r\right)\right) \varphi(\vec{x}, \vec{y}), \quad i, j=1,2, \\
& \bigwedge_{n \geq 1} \bigvee_{m \geq 1}\left(P_{i} \vec{y}<\frac{1}{n}\right)\left(P_{j} \vec{x} \in\left(r, r+\frac{1}{m}\right]\right) \varphi(\vec{x}, \vec{y}) .
\end{aligned}
$$

Received by the editors February 19, 1986.

1980 Mathematics Subject Classification (1985 Revision). Primary 03C70; Secondary 03H10. 
Axioms of singularity.

$$
\left(P_{i} x=0\right)\left(\left(P_{1} y>0\right)(x=y) \wedge\left(P_{2} y>0\right)(x=y)\right), \quad i=1,2 .
$$

In order to prove the main result, let us introduce two sorts of auxiliary models.

DEFINITION. (i) A weak structure for $L_{P_{1} P_{2} \mathfrak{A}}$ is a structure $\left(\mathfrak{A}, \mu_{n, i}\right)_{n \geq 1, i=1,2}$ (shortly $\left(\mathfrak{A}, \mu_{n, i}\right)$ ) such that each $\mu_{n, i}$ is a finitely additive probability measure on $A^{n}$ with each singleton measurable and the set $\left\{\vec{b} \in A^{n}:\left(\mathfrak{A}, \mu_{n, i}\right) \vDash \varphi[\vec{a}, \vec{b}]\right\}$ is $\mu_{n, i}$-measurable for each $\varphi(\vec{x}, \vec{y}) \in L_{P_{1} P_{2} A}$ and $\vec{a} \in A^{m}$.

(ii) A middle structure for $L_{P_{1} P_{2} A}$ is a weak structure $\left(\mathfrak{A}, \mu_{n, i}\right)$ such that the following is true:

There is a set $B \subseteq A$ such that $\mu_{1}(B)=0$ and $\mu_{2}(B)=1$.

In both cases the concept of satisfaction is defined in a natural way.

LEMMA 1. A theory $T$ of $L_{P_{1} P_{2} A}$ is consistent if and only if it has a weak model in which each theorem of $L_{P_{1} P_{2} A}$ is true.

The proof involves the Henkin construction similar to that in [1].

In order to prove the next lemma, we use the following Los-Marczewski theorem.

THEOREM 1 (SEE [4], [5]). Let $C$ be a field of subsets of a set $\Omega$. Let $\mu$ be a finitely additive probability measure on $C$. Let $A \subseteq \Omega$ be such that $A \notin C$. Let $\mathcal{F}(C, A)$ be the smallest field on $\Omega$ containing $C$ and $\bar{A}$. Then there exists a finitely additive probability measure $\bar{\mu}$ on $\mathcal{F}(C, A)$ which is an extension of $\mu$.

Moreover, if $d$ is any real number between

$$
\begin{array}{r}
\mu_{i}(A)=\sup \left\{\frac{\sum_{j=1}^{n} \mu\left(A_{j}\right)-\sum_{j=1}^{m} \mu\left(B_{j}\right)}{k}: A_{1}, \ldots, A_{n}, B_{1}, \ldots, B_{m},\right. \\
\left.k I_{A}+\sum_{j=1}^{m} I_{B_{j}} \geq \sum_{j=1}^{n} I_{A_{i}}\right\}
\end{array}
$$

and

$$
\begin{array}{r}
\mu_{e}(A)=\inf \left\{\frac{\sum_{j=1}^{n} \mu\left(A_{j}\right)-\sum_{j=1}^{m} \mu\left(B_{j}\right)}{k}: A_{1}, \ldots, A_{n}, B_{1}, \ldots, B_{m},\right. \\
\left.\sum_{j=1}^{n} I_{A_{j}} \geq \sum_{j=1}^{m} I_{B_{j}}+k I_{A}\right\},
\end{array}
$$

then there is a finitely additive probability measure $\bar{\mu}$ on $\mathcal{F}(C, A)$ such that $\bar{\mu}(A)=d$ and $\bar{\mu}$ is an extension of $\mu$ from $C$ to $\mathcal{F}(C, A)$. ( $I_{A}$ is an indicator function of the set $A$.)

LEMMA 2. A theory $T$ of $L_{P_{1} P_{2} A}$ is consistent if and only if it has a middle model in which each theorem of $L_{P_{1} P_{2} A}$ is true.

Proof. The nontrivial part is to prove that if $T$ is consistent then $T$ has a middle model.

Let $\left(\mathfrak{A}, \mu_{n, i}\right)$ be a weak model of $T$ in which each theorem of $L_{P_{1} P_{2} \mathfrak{A}}$ is true.

Let $Z_{0}, Z_{1}, \ldots$ enumerate $\operatorname{dom} \mu_{1,1}=\operatorname{dom} \mu_{1,2}$. 
Let us choose sets $U$ and $S$ in the following way:

$$
\begin{aligned}
& U_{0}=\left\{x \in A: \mu_{1,1}(\{x\})>0\right\}, \quad S_{0}=\left\{x \in A: \mu_{1,2}(\{x\})>0\right\}, \\
& U_{n+1}=U_{n} \cup\{x\}, \quad \text { where } x \in Z_{n} \backslash\left(U_{n} \cup S_{n}\right) \quad \text { (if there is any), } \\
& S_{n+1}=S_{n} \cup\{x\}, \quad \text { where } x \in Z_{n} \backslash\left(U_{n+1} \cup S_{n}\right) \quad \text { (if there is any). }
\end{aligned}
$$

Let $U=\bigcup_{n \geq 0} U_{n}$ and $S=\bigcup_{n \geq 0} S_{n}$. It is easy to see that $U \cap S=\varnothing$ and that each set $Z_{n} \in \operatorname{dom} \mu_{1,1}=\operatorname{dom} \mu_{1,2}$ with a positive measure $\mu_{1,1}\left(\mu_{1,2}\right)$ has a nonempty intersection with $U\left(U^{c}\right)$.

Let us show that $\left(\mu_{1,1}\right)_{e}(U)=1$. (Let us abbreviate $\left(\mu_{1,1}\right)_{e}$ as $\mu$ in the rest of the proof.)

Let $A_{1}, \ldots, A_{n}, B_{1}, \ldots B_{m}$, be members from dom $\mu$ such that

$$
\sum_{i=1}^{n} I_{A_{i}} \geq \sum_{i=1}^{m} I_{B_{i}}+k I_{A} .
$$

Let $C_{s}=\left\{x \in A: \sum_{i=1}^{n} I_{A_{i}}(x)=s\right\}, s=0, \ldots, n, D_{s}=\left\{x \in A: \sum_{i=1}^{m} I_{B_{i}}(x)\right.$ $=s\}, s=0, \ldots, m$.

It is easy to see that $\mu\left(D_{s}\right)=0$ for $s>n-k$ and $\mu\left(C_{s}\right)=0$ for $s<k$. Consequently $\mu\left(D_{n-k}\right)+\cdots+\mu\left(D_{n-k-i}\right) \leq \mu\left(C_{n}\right)+\cdots+\mu\left(C_{n-i}\right)$ for $i=0, \ldots, n$, $k-1$.

It follows that

$$
\begin{aligned}
\sum_{i=1}^{n} \mu & \left(A_{i}\right)-\sum_{i=1}^{m} \mu\left(B_{i}\right)=\sum_{s=1}^{n} s \mu\left(C_{s}\right)-\sum_{s=1}^{m} s \mu\left(D_{s}\right) \\
& =\sum_{s=k}^{n} s \mu\left(C_{s}\right)-\sum_{s=1}^{n-k} s \mu\left(D_{s}\right)=\sum_{s=k}^{n} s \mu\left(C_{s}\right)-\sum_{s=k}^{n}(s-k) \mu\left(D_{s-k}\right) \\
& \geq \sum_{s=k}^{n} s \mu\left(C_{s}\right)-\sum_{s=k}^{n}(s-k) \mu\left(C_{s}\right)=\sum_{s=k}^{n} k \mu\left(C_{s}\right)=k \sum_{s=0}^{n} \mu\left(C_{s}\right)=k .
\end{aligned}
$$

So, $\mu_{e}(U)=1$ or in precise notation $\left(\mu_{1,1}\right)_{e}(U)=1$.

Similarly it can be shown that $\left(\mu_{1,2}\right)_{e}\left(U^{c}\right)=1$.

Hence by Theorem 1 , measures $\mu_{1,1}$ and $\mu_{1,2}$ can be extended so that $\mu_{1,1} \subseteq \bar{\mu}_{1,1}$, $\mu_{1,2} \subseteq \bar{\mu}_{1,2}$ and $\bar{\mu}_{1,1} \perp \bar{\mu}_{1,2}$.

LEMMA 3. For each weak model $\left(\mathfrak{A}, \mu_{n, i}\right)$ in which each theorem of $L_{P_{1} P_{2} A}$ is true there is a singluar biprobability model $\left(\mathfrak{A}, \mu_{1}, \mu_{2}\right)$ such that $\left(\mathfrak{A}, \mu_{n, i}\right) \equiv$ $\left(\mathfrak{A}, \mu_{1}, \mu_{2}\right)$.

The proof of the lemma makes use of Loeb-Hoover-Keisler construction (see [3]) and Lemma 2.

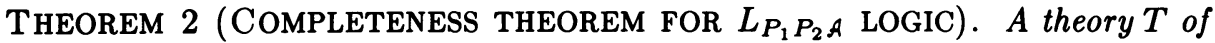
$L_{P_{1} P_{2} A}$ is consistent if and only if $T$ has a singular biprobability model.

The proof follows easily from Lemmas 1,2 and 3. 


\section{BIBLIOGRAPHY}

1. D. Hoover, Probability logic, Ann. Math. Logic 14 (1978), 287-313.

2. H. J. Keisler, Hyperfinite model theory, Logic Colloquium '76, edited by J. M. E. Hyland and R. O. Gandy, North-Holland, 1977, pp. 5-110.

3. _ Probability quantifiers, Chapter 14 in Model Theoretic Logics, edited by J. Barwise and S. Feferman, Springer-Verlag, 1985, pp. 509-556.

4. J. Los and E. Marczewski, Extension of measures, Fund. Math. 36 (1949), 267-276.

5. K. P. S. Rao Bhaskara and M. Rao Bhaskara, Theory of charges, Academic Press, 1983.

6. M. D. Raškovic, Completeness theorem for biprobability models, J. Symbolic Logic 51 (1986), 586-590.

Prirodno-Matematiči faK., Radoja Domanovića 12, 34000 KragujeVaC, YuGOSLAVIA

Matematič́i Institut, Kneza Mihajla 35, 11000 Beograd, Yugoslavia (Current address) 